\title{
EXPERIMENTS OF CLOSED-LOOP FLOW CONTROL FOR LAMINAR BOUNDARY LAYERS
}

\author{
ZHENYU ZHANG \\ Department of Aerodynamics, College of Aerospace Engineering \\ Nanjing University of Aeronautics and Astronautics, Yudao Street 29 \\ Nanjing, 210016, China \\ zyzhang@nuaa.edu.cn
}

\begin{abstract}
The Tollmien-Schlichting (T-S) instability is important for the transition process of boundary layer flows. The cancellation of its unstable modes will help delay the occurrence of transition and then turbulence. In this article the T-S instability was attempted to attenuate through an active closed-loop control strategy. The frequency responses of unstable travelling T-S waves were obtained for the implementation of control strategy. Information such as velocity disturbances of both incoming unstable waves and cancelling waves were then fed into the control system. A controller was deliberately designed and tested for the active cancellation of instabilities which were excited by velocity disturbances normal to the wall. The cancellation performance of the control system was validated by experiments in a low turbulence wind tunnel, which proved the functionality of above control strategy upon the active cancellation of boundary layer instabilities.
\end{abstract}

Keywords: T-S instability; closed-loop control; laminar boundary layers.

\section{Introduction}

It is well known that the skin friction due to a turbulent boundary layer may be greater than the one at laminar state within considerable range of Reynolds number ${ }^{1}$. Therefore, a laminar boundary layer is generally favorable for researches like drag reduction. A possible route towards this objective is to extend the laminar area by delaying the onset of transition to turbulence.

At the early stage of transition, the Tollmien-Schlichting (T-S) instability is commonly seen in a boundary layer for quasi-parallel flow, such as the one along a flat plate. According to the hydrodynamic instability theory, small disturbances in the boundary layer may be amplified during the propagation to downstream, if both their frequencies and wave numbers fall into the unstable domain which is determined by the dispersion relation of the Orr-Sommerfeld equation. Then the exponential growth of certain unstable modes may be followed by nonlinear interactions between these modes when their magnitudes get considerable, which cause the transition occur quickly. Therefore a delay of transition may be realized by eliminating or attenuating the magnitudes of unstable modes in the boundary layer. This objective will be accomplished, if the initial linear modes, say, the T-S instabilities, can be attenuated to a low enough level through deliberately designed control strategies. 


\section{Control of T-S Instabilities}

Researches on the control of T-S instabilities can be surveyed back to the validating experiments by both Schubauer and Skramstad ${ }^{2}$ at National Bureau of Standards of United States in 1943, in which the T-S modes were excited in a flat-plate boundary layer by an electrically driven vibrating ribbon. In their experiments, the instabilities move downstream as periodically travelling waves with the driven frequency of the ribbon, which showed a stream-wise amplification for certain band of wave numbers. Their experiments validated the prediction results from both Tollmien and Schlichting ${ }^{1}$.

In the early 1980s, researchers focused more upon the cancellation of unstable T-S waves for the delay of transition, and some control strategies were introduced on the basis of the "active wave cancellation" concept. The main idea of them is that the T-S instabilities are essentially linear waves, and then their magnitudes may be significantly reduced by linear superimposition with other out-of-phase cancelling waves. Milling ${ }^{3}$ reported the rise of critical Reynolds number in a boundary layer water flow along a flat plate by driving a vibrating wire with out-of-phase signals. Further improvements were also found from research of Liepmann et $a .^{4}{ }^{4}$ using heating strips. The wind tunnel experiment from Thomas ${ }^{5}$ showed that the transition due to both single frequency amplification and nonlinear interaction between distinctive waves were controllable by active cancellation methods.

In the recent decades, more researchers suggested that if the unstable disturbances were detected by sensors and their signal were fed forward into a control system to generate the cancelling waves downstream, the magnitudes of unstable modes would be attenuated for delay of transition. Successes were observed in the researches such as Pupator and Saric $^{6}$ and Joslin $^{7}$, respectively. Pupator and Saric investigated the cancellation of random noises in boundary layer flow. In Joslin's report, a spectral controller could be designed for more than $60 \%$ cancellation. Further progresses of T-S wave cancellation on the surface of an unswept wing have been reported by Sturzebecher and Nitsche ${ }^{8}$ with an adaptive controller. Experiments of $\mathrm{Li}^{9}$ showed $80 \%$ cancellation by an open loop controller for the T-S instability cancellation in a fat plate boundary layer. The controller was designed according to the transfer functions of the unstable T-S modes.

Nearly all the above control strategies can be considered open loop schemes. Disadvantages of these strategies may appear under off-designed conditions, such as weak resistance against background noises. Part of them was discussed in Li's thesis . Therefore, a closed-loop strategy may be favourable over the open loop ones at these aspects.

\section{The Design of Closed-loop Control Scheme}

The closed-loop cancellation scheme of T-S instabilities in a flat-plate boundary layer flow can be implemented as shown in Fig. 1. The control system consists of sensors, controllers and actuators upstream to the sensor. 


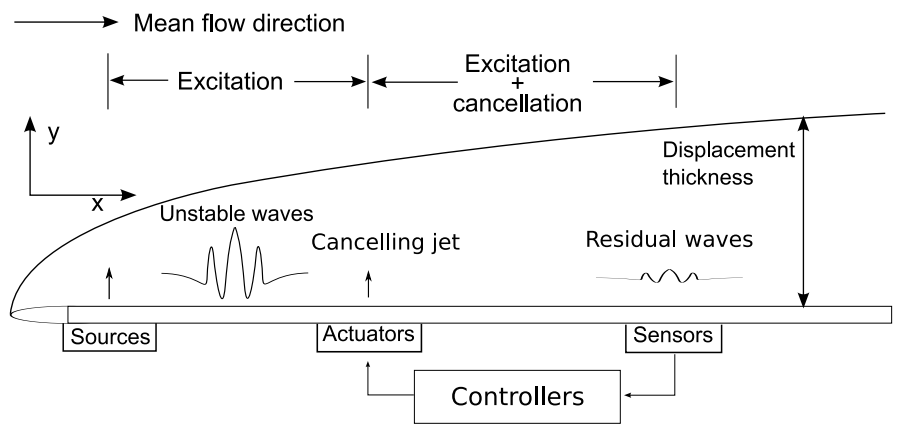

Fig. 1. The Scheme of closed-loop wave cancellation for T-S instabilities.

Under lab condition, the unstable T-S waves can be excited by a point velocity disturbance source far upstream. Their amplified modes during the convective propagation are supposed to be cancelled by the out-of-phase cancelling waves downstream. The resultant magnitudes are to be minimized by the control system at the positions of sensors.

\section{Experimental Configuration}

The experiments were conducted in the 6-feet long, 3 feet by 3 feet test section of a low turbulence wind tunnel of Queen Mary, University of London. A 65-inch aluminium flat plate was mounted at the centre of the test section. The effects of stream-wise pressure gradient were removed by deliberately adjusting the following two-element flap.

The coordinate system was set up as follows: The $x$-axis starts from the leading edge of plate and follows the streamwise direction; The $y$-axis is perpendicular to the plate surface; the $z$-axis is along the spanwise direction according to right-handed system convention.

The T-S instabilities were excited by the normal jet disturbances at $x=200 \mathrm{~mm}$ from the leading edge of the plate. The jet was formed through a $1 \mathrm{~mm}$ hole by a $12 \mathrm{~mm}$ minispeaker underneath the plate. The driving signal for the disturbance source comes from a D/A module of a Microlink data acquisition system. In current experiments the signals are chosen as both sinusoidal and impulse function, respectively.

The control system is composed of one hot-wire probe as sensor, a low-order operational amplifier-based controller and a twin-slot jet actuator. Both the actuator and sensor are mounted in a $180 \mathrm{~mm}$ diameter aluminium round disk which is placed at $x=530 \mathrm{~mm}$ from the leading edge. The $1 \mathrm{~mm}$ wide, $10 \mathrm{~mm}$ long slot actuator was set at $x=500 \mathrm{~mm}$. The twin slots have a $3 \mathrm{~mm}$ interval for avoiding their unfavorable near-field effect on the cancellation. The sensor is at $x=529 \mathrm{~mm}$ and $z=0 \mathrm{~mm}$. The set-up of both actuator and sensor are shown in Fig. 2. 


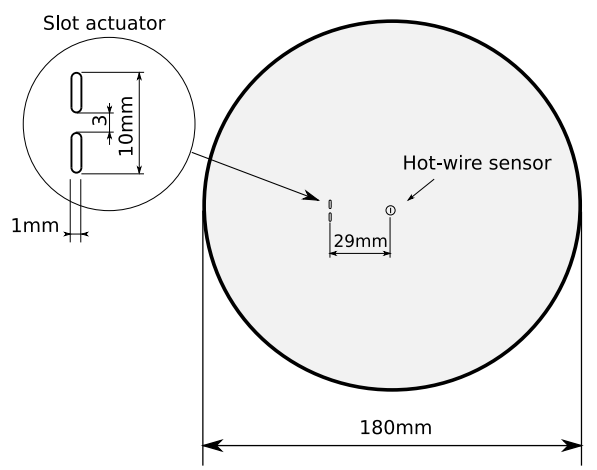

Fig. 2. The actuator and hot-wire sensor on the round plate.

\section{Design of Controller}

The convective essence of T-S instability makes the frequency band of the unstable wave modes very narrow. With the streamwise propagation, the central peak of the instabilties shift to lower frequency. In order to cancel the unstable T-S waves upstream by a closedloop control scheme, both the frequency response of the plant in the loop and the stability of whole closed-loop system must be examined, respectively.

With current experimental set up, at the position of sensor, the local Reynolds number is $\operatorname{Re}_{\delta *}=1204$. The unstable waves show a frequency peak near $1300 \mathrm{rad} / \mathrm{s}$, which is actually neutral at that location. Since from the sensor's position only the amplifying waves below the peak frequency will keep growing downstream, the central frequency band of the controller will be adjusted accordingly to 600-1300 rad/s, as shown in Fig. 3 .

The controller was implemented based on the following transfer function

$$
\frac{-60(s+1225)\left(s^{2}+2100 s+3.5 e 6\right)}{\left(s^{2}+650 s+6.6 e 5\right)\left(s^{2}+860 s+1.5 e 6\right)} \text {. }
$$

Before the cancellation experiments, the frequency response of the cancelling wave from actuator were acquired for the stability analysis of closed-loop control system. The cancelling waves from the actuator were measured by the sensor at $x=529 \mathrm{~mm}, \eta=0.5$ and $z=0 \mathrm{~mm}$. The magnitude response versus frequency of cancelling wave is shown by the Bode plot in Fig. 4. Here the non-dimensional height $\eta$ comes from the definition

$$
\eta=y \sqrt{\frac{U_{\infty}}{v x}} .
$$

where $x, y, U_{\infty}$ and $v$ are the stream-wise, normal coordinates, freestream velocity and viscosity, respectively.

With the above arrangement, current control system is stable according to the Nyquist criterion for the minimization of the incoming unstable T-S modes. Here the influence of cancelling waves, responses from both controller and hot-wire sensor, are all considered. 

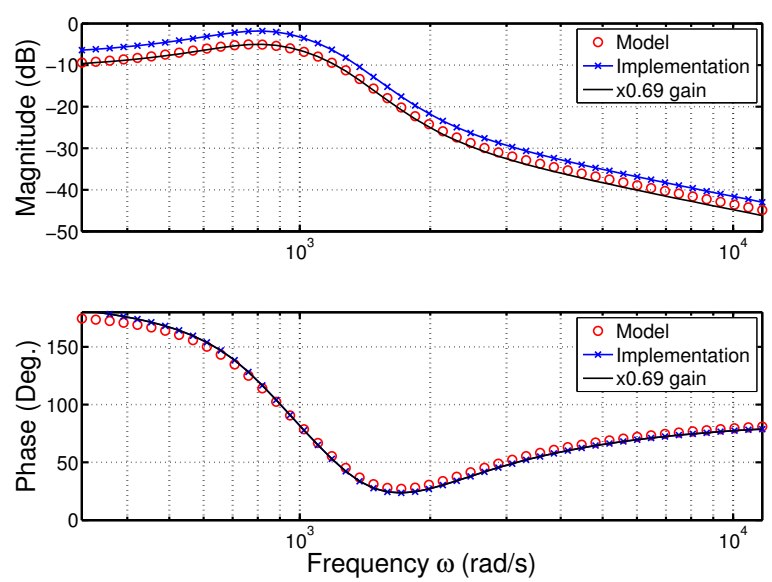

Fig. 3. Frequency and phase spectra of the controller.

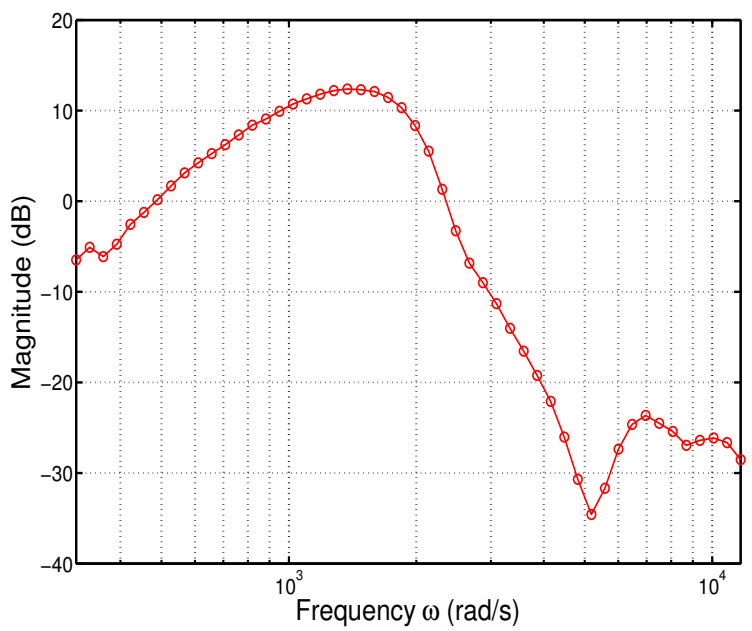

Fig. 4. Frequency response of the cancelling wave.

\section{Results of Wave Cancellation Experiments}

\subsection{Tests for the cancellation of impulse excited instabilities}

Since the behavior of a linear system can be determined by its impulse response, the performance of the above closed-loop control system was tested by wind tunnel experiments for the cancellation of unstable T-S waves excited by impulsive velocity disturbances.

With free stream speed of $15 \mathrm{~m} / \mathrm{s}$, and the Reynolds number of $\operatorname{Re}_{\delta *}=1204$, the cancellation of impulse excited T-S waves were measured by another hot-wire probe 
traversing downstream of the control system, ranging from 550 to $750 \mathrm{~mm}$ in the $x$ direction and -50 to $50 \mathrm{~mm}$ in the $z$-direction. The height of the probe over the plate is set to $\eta=0.5$. The impulsive velocity disturbance comes from the signal generator of the Microlink system. The width and period of the impulse signal are $3.125 \mathrm{~ms}$ and $0.2 \mathrm{~s}$, respectively. For each sample the length is also $0.2 \mathrm{~s}$ and 256 points are contained. Then the sampling frequency is $256 / 0.2=1280 \mathrm{~Hz}$. Every 128 samples were grouped for the averaging.

Fig. 5 shows the spanwise distributions of the streamwise velocity disturbances in $r m s$ value which were measured about $200 \mathrm{~mm}$ downstream of the control system. The averaged time histories results are shown in Fig. 6. By comparing the uncontrolled instabilities and their controlled results, reduction towards both rms values and magnitudes of the stremawise velocity disturbances were observed in Figs. 5 and 6, respectively. The decrease of $r m s$ value is up to $30 \%$ near the central position, which means a 50\% reduction in the energy of the T-S instabilities. Such a decrease can also be found from the comparison of time histories in Fig. 6. The attenuation effects of the current control system are attributed to the cancellation of the developing modes below the peak frequency. Since those modes within higher frequency range have positive imaginary parts in their wave number and then will naturally damp out downstream, they will not be covered by the central band of the closed-loop system. Moreover, the time histories showed the cancellation was not significant until the second peak was reached. This can be explained by the genuine time delay due to the convection from the actuator to the sensor. Only when the second peak reaches the position of actuator, the sensor begins to catch the front of the travelling wave packet and then drive the controller to work. Therefore, a shorter distance between the two elements in the control system seems preferable in this case.

\subsection{The resistance of control system against external noises}

In order to check the noise resistance property, the above control system was also tested for the cancellation of T-S waves driven by banded signal with distinctive noise levels. The excitation frequencies were banded between 100 and $200 \mathrm{~Hz}$. The phase angles were randomly distributed between -180 to 180 degree. These signals were combined with noises with signal-to-noise ratios (SNR) of 100, 10, 5 and 2, respectively. Disturbances excited by these signals were measured by the traversing hot-wire probe at $x=750 \mathrm{~mm}$. The rms results normalized by the freestream speed indicate quite steady cancellation around $70 \%$ for all four noise levels, as seen in Table 1. Results from the open loop controller by $\mathrm{Li}^{9}$ indicated that there were $30-43 \%$ variations of cancellation with respect to SNR of 1 to 10. But in the present study, the closed-loop control system showed more tolerance against external noises, which is advantageous due to the closed-loop arrangements. 


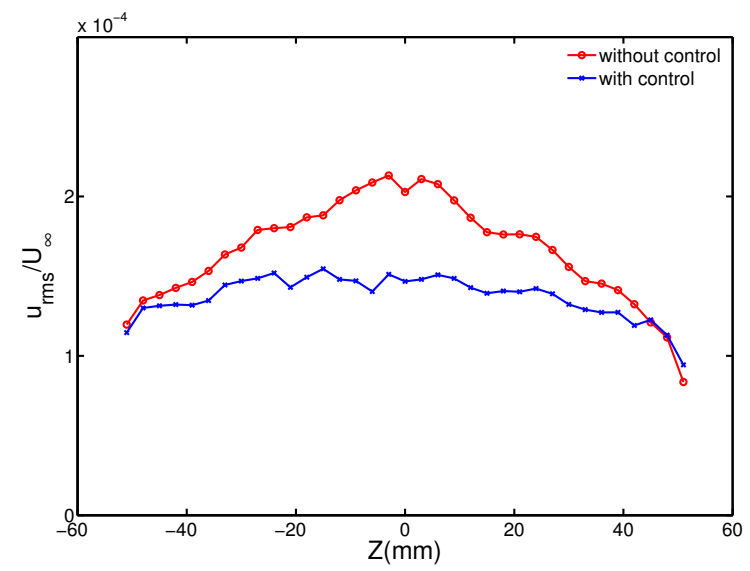

Fig. 5. Spanwise $r m s$ distribution of the streamwise velocity disturbances measured at $x=750 \mathrm{~mm}$ and $\eta=0.5$.

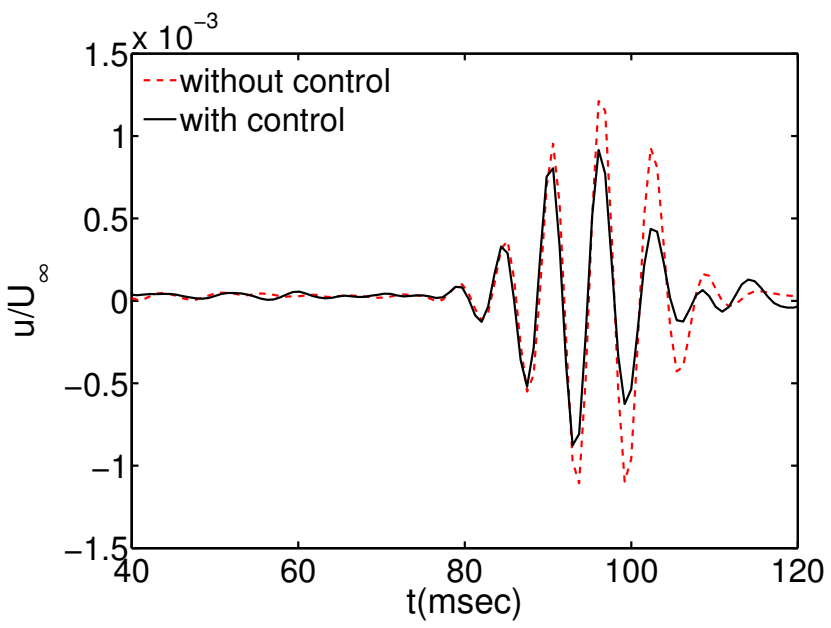

Fig. 6. Time history of the streamwise velocity disturbances measured at $x=750 \mathrm{~mm}$ and $\eta=0.5$.

Table 1. Normalized $r m s$ value of streramwise velocity disturbances at $x=750 \mathrm{~mm}$ for four SNR levels.

\begin{tabular}{l|l|l|l|l}
\hline SNR & 100 & 10 & 5 & 2 \\
\hline$r m s$ & 0.744 & 0.714 & 0.717 & 0.713 \\
\hline
\end{tabular}

\section{Conclusions}

The linear hydrodynamic instability theory states that in a boundary layer flow the Tollmien-Schlichting waves with unstable frequencies and wave numbers will be amplified when propagating in the stream-wise direction. Through a well designed 
closed-loop control system, the magnitude of the unstable waves may be significantly decreased for the delay of transition. In comparison with open loop control schemes, a closed-loop strategy will not be vulnerable to the contamination from external noises within certain frequency range. However, from the viewpoint of dynamic system, the rapid phase shift of the control system within a narrow frequency band indicates a high dimension nature of the boundary layer flow, which makes the stability of closed-loop control system a critical requirement for its application. Therefore, further efforts on the applications of closed-loop cancellation should be exerted more to the design of distributed arrays of control units for which a multi-input, multi-output flow control model will be considered in the near future.

\section{Acknowledgments}

The author would like thank Prof. M. Gaster both for the support to current study and for his constructive suggestions and arguments. The author also wants to thank Dr Y. Li for active communications and discussions on this research.

\section{References}

1. H. Schlichting and K. Gersten, Boundary-Layer Theory, 8nd edn. (Springer-Verlag, Berlin, 2000).

2. G. B. Schubauer and H. K. Skramstad, J. Res. Natl. Bur. Stand. 38, 251 (1947).

3. R. Milling, Phys. Fluids, 24, 979 (1981).

4. H. W. Liepmann, G. L. Brown and D. M. Nosenchuck, J. Fluid Mech. 118, 187 (1982).

5. A. S. W. Thomas, J. Fluid Mech. 137, 233 (1983).

6. P. Pupator and W. Saric, AIAA paper, 89-1007, 1989.

7. R. D. Joslin, G. Erlebacher and M. Y. Hussaini, J. Fluid Eng. 118, 494 (1996).

8. D. Sturzebecher and W. Nitsche, Special Iss. Int. J. Heat Fluid Flow, 24, 572 (2003).

9. Y. Li, Active control of boundary layer instabilities, $\mathrm{PhD}$ thesis, (2004). 